\title{
AUTONOMOUS NAVIGATION AND ATTITUDE CONTROL OF SPACECRAFTS ON NEAR-EARTH CIRCULAR ORBITS
}

\author{
Andronov Vladimir Germanovitch* Emelyanov Sergey Gennadievitch \\ Southwest State University, Kursk, Russia
}

The paper reviews existing methods of spacecraft navigation and attitude control on near-earth orbits, including the methods utilizing earth-based external trajectory measurement stations, global navigation satellite systems, inertial on-board navigation systems and star trackers. The proposed method is based on the obtaining and processing of a set of sky images on-board of a spacecraft that is moving along a near-earth circular orbit. Image processing results are used to find the coordinates, velocity and attitude of a spacecraft in inertial space.

Key words: Star tracker, Autonomous navigation of spacecrafts, Navigation

\section{INTRODUCTION}

To control a spacecraft (SC) efficiently the operator should have an opportunity to determine its attitude, velocity and the orientation of structural axes in inertial space continuously [01], and this is the essence of navigation and attitude control tasks. Until recently the methods of a SC navigation and attitude control have been based on external tracking that used to be done through a network of ground observation stations and services by means of astrometric, radio-technical or laser monitoring of the SC. Today the efficiency of such external tracking systems has degraded significantly due to a sharp growth of the number of SC s on different orbits. This challenge has been successfully met by global navigation satellite systems (GNSS) that are able to measure coordinates and velocities of vehicles, whether on the Earth surface or in near-earth space, with high accuracy and efficiency. Moreover, GNSS performance is invariable irrelevantly of the quantity of SCs or other objects of control that use GNSS data.

However, as the space exploration is extending the development of the methods and means of SC autonomous navigation and attitude control that will be based on the data that are available on board of a SC, or can be obtained by SC self-systems, or by means of applying natural reference points, has become an even more important objective. All methods of SC autonomous navigation and/or attitude control can be divided into three groups. The first group of methods is based on inertial systems of navigation and attitude control [02]. In these systems different types of gyroscopes are used. At first those were mechanical gyroscopes. In these devices the axis of rotation is unaffected by tilting or rotation of the mounting and because of this they can be used for reckoning and orientation with a high degree of accuracy. Modern gyroscopes (laser, optical fiber or micromechanical devices) are used to measure velocity and rotational acceleration about a fixed axis. These new gyroscopes are space-effective and lighter in weight compared with mechanical ones, while their accuracy is steadily approaching that of mechanical instruments. However, inertial navigation and orientation systems have one ineradicable fault, the so called "drift, or gyroscope wander", which is inherent in all gyroscope types and is caused by random unforeseeable factors that can affect the gyroscope performance. For example, the drift of a mechanical gyroscope is caused by random forces affecting the gyroscope support bearings. The drift rate can hardly be closely predicted; however, it can be estimated, if there are several gyroscopes available. The drift in other gyroscope types may be caused by other factors, yet all of them produce similar effect. The drift rate is increasing with time, usually proportional to , and within a certain time period the precision of the data generated by an autonomous system degrades inadmissibly. In order to remedy the error inertial navigation and orientation systems with continued operation are regularly tested to match the measurements obtained by an inertial system with the data of GNSS and/or astrometric systems that use stellar trackers (STs).

The second group of the methods of SC autonomous navigation and orientation are based on the use of stars and/or the Earth as external natural reference points. If stars are used, a sky area image is made from the board of a SC. The image is processed to recognize those stars that are registered in the on-board star catalogue. After that their known inertial coordinates are applied for self-production of the cosine matrix that describes the orientation of the ST coordinate system and the SC structural axes in inertial space. As stars are infinitely remote objects, their position in the inertial coordinate system remains unchanged, so the use of stars as external reference points ensures pinpoint accuracy of the SC attitude measurements, which today is several arcseconds and potentially can be just arcsecond fractions.

The spacecraft earth attitude determination in inertial space is actually limited to finding the Earth center line in the system of coordinates fixed with a specific gyro-sensor and translating this line into SC-fixed system of struc- 
tural axes coordinates. This process is called the determination of vertical geo-reference. Irrespective of the ways of vertical geo-reference determination the main problem here is big angular size of the Earth, which can amount $110^{\circ} \mathrm{C}$, if a SC is on a near-earth orbit. It causes serious measurement errors that can be up to a degree tenth [03]. An essential disadvantage of all existing methods that utilize separate use of stars and the Earth as external references is the possibility to determine only the SC attitude.

The third group of the methods of SC autonomous navigation and orientation in the near-earth space is related with an integrated use of stars and the Earth reference as it permits determining both the SC attitude and its coordinates in inertial space autonomously [04]. At the same time inadequate accuracy of vertical geo-reference determination tells on the navigation accuracy making it insufficient to meet many objectives of the Earth remote sensing from the space.

\section{A METHOD OF SPACECRAFT AUTONOMOUS NAVIGATION AND ATTITUDE CONTROL ON A CIRCULAR ORBIT}

In this paper we introduce a method of solving the problem of precise autonomous navigation and attitude control of a spacecraft on a circular orbit. The method is based on the results of processing sky images. The problem shall be set up as follows.

Let a SC be in motion along in a circular orbit and at a time moment $t$ be located in a point $S$ in the inertial space (Figure 1) formed up by inertial coordinate system (ICS)

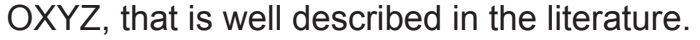

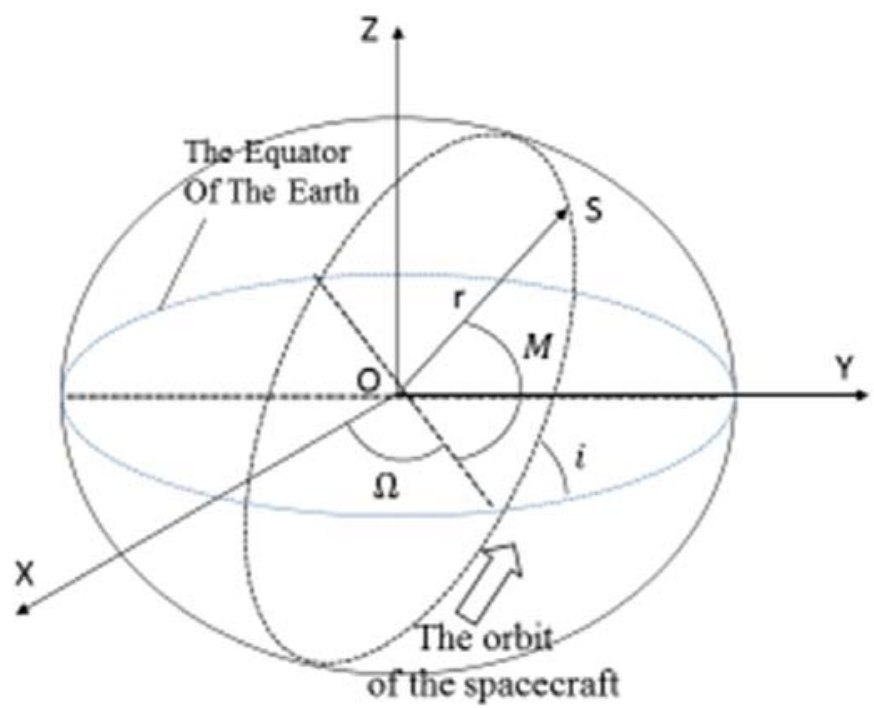

Figure 1: To define the parameters of a circular orbit
As for a circular orbit the following relationships hold true $a=r ; e=0 ; M(t)=E(t)=\vartheta(t)=u(t)$, where $a, e$ - are the orbit semimajor axis and eccentricity; $r$ - SC R-bar; $M(t), E(t)$ and $\vartheta(t)$ - are SC mean, eccentric and true anomalies; and $u(t)$ - is argument of latitude, the position of the spacecraft in any time moment $t$ will be entirely determined by its R-bar $r$, inclination angle $i$, longitude of ascending node $\Omega$ and mean anomaly $\mathrm{M}(t)$.

Now let's assume that on SC board there is a star tracker having linear eccentricity $f$, and at a certain time moment $t$ this tracker makes a sky image and this image has been processed for automatic recognition of * 1 and *2 stars that a priori have known inertial coordinates $\left(\alpha_{1}, \delta_{1}\right),\left(\alpha_{2}, \delta_{2}\right)$ in ICS, while their plane coordinates $\left(x_{1}^{*}, y_{1}^{*}\right),\left(x_{2}^{*}, y_{2}^{*}\right)$ have been measured in the image coordinate system. It is necessary to find the elements of the SC keplerian orbit $r, \mathrm{M}(t), i, \Omega, \mathrm{SC}$ coordinates and velocity components at the time moment $t$.

The main point in the problem solution (Figure 2) is to find the direction of the axis of homology OS of planes OSK and OSJ formed up by stellar lines SK and OK of the star *1, and lines $S J$ and $O J$ of the star *2. Let's find unit vectors $\left(\boldsymbol{l}_{1}, \boldsymbol{l}_{2}\right)$ and $\left(\boldsymbol{L}_{1}, \boldsymbol{L}_{2}\right)$.

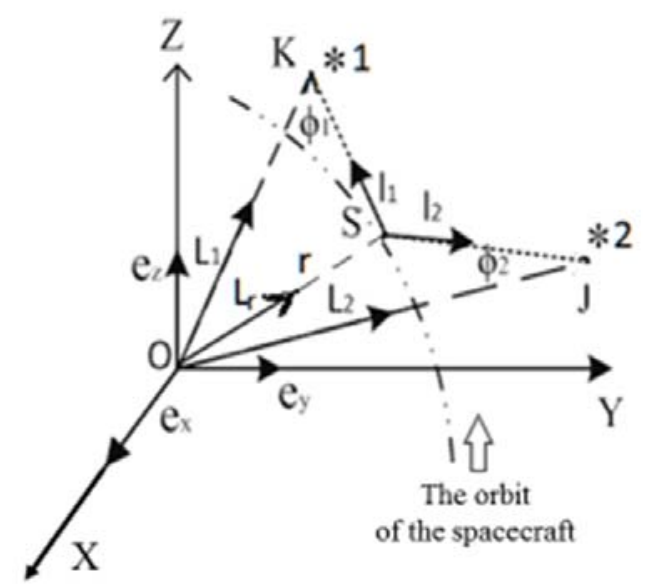

Figure 2: Finding the direction of the radius vector of the spacecraft

$$
\begin{aligned}
& \boldsymbol{l}_{\mathbf{1}}=\left(l_{x}^{(1)}, l_{y}^{(1)}, l_{z}^{(1)}\right)^{\mathrm{T}}=\left(\begin{array}{l}
x_{1}^{*} / \Delta_{1} \\
y_{1}^{*} / \Delta_{1} \\
f^{*} / \Delta_{1}
\end{array}\right) \\
& \boldsymbol{l}_{2}=\left(l_{x}^{(2)}, l_{y}^{(2)}, l_{z}^{(2)}\right)^{\mathrm{T}}=\left(\begin{array}{l}
x_{2}^{*} / \Delta_{2} \\
y_{2}^{*} / \Delta_{2} \\
f^{*} / \Delta_{2}
\end{array}\right) \\
& \boldsymbol{L}_{1}=\left(L_{x}^{(1)}, L_{Y}^{(1)}, L_{z}^{(1)}\right)^{\mathrm{T}}= \\
& =\left(\cos \alpha_{1} \cdot \cos \delta_{1}, \sin \alpha_{1} \cdot \cos \delta_{1}, \sin \delta_{1}\right)^{\mathrm{T}} \\
& \Delta_{1}=\sqrt{\left(x_{1}^{*}\right)^{2}+\left(y_{1}^{*}\right)^{2}+\left(f^{*}\right)^{2}} \\
& \Delta_{2}=\sqrt{\left(x_{2}^{*}\right)^{2}+\left(y_{2}^{*}\right)^{2}+\left(f^{*}\right)^{2}}
\end{aligned}
$$


As is known in the theory of extraterrestrial photogrammetry the unit vector of the axis of homology is vector product of the vector products of the unit vectors that form the planes.

Then unit vector $\boldsymbol{L}_{r}(t)$ can be described in normal orthogonal basis $\mathrm{e}_{x}, \mathrm{e}_{\boldsymbol{y}}, \mathrm{e}_{z}$ as follows:

$$
\begin{aligned}
& \boldsymbol{L}_{r}(t)=\left[N_{1}(t) \times N_{2}(t)\right]= \\
& =\left[\left(\boldsymbol{l}_{1} \times \boldsymbol{L}_{\mathbf{1}}\right] \times\left[\left(\boldsymbol{l}_{2} \times \boldsymbol{L}_{2}\right]\right.\right. \\
& \boldsymbol{N}_{\mathbf{1}}(t)=\left(\begin{array}{l}
N_{X}^{(1)}(t) \\
N_{Y}^{(1)}(t) \\
N_{Z}^{(1)}(t)
\end{array}\right)=\frac{1}{\sin \emptyset_{1}} \cdot\left|\begin{array}{ccc}
\mathrm{e}_{X} & \mathrm{e}_{Y} & \mathrm{e}_{Z} \\
l_{x}^{(1)} & l_{y}^{(1)} & l_{z}^{(1)} \\
L_{X}^{(1)} & L_{Y}^{(1)} & L_{Z}^{(1)}
\end{array}\right|= \\
& =\frac{1}{\sin \emptyset_{1}} \cdot\left(\begin{array}{l}
\bar{N}_{X}^{(1)} \\
\bar{N}_{Y}^{(1)} \\
\bar{N}_{Z}^{(1)}
\end{array}\right)
\end{aligned}
$$

where:

$$
\begin{aligned}
& \left(\begin{array}{l}
\bar{N}_{X}^{(1)} \\
\bar{N}_{Y}^{(1)} \\
\bar{N}_{Z}^{(1)}
\end{array}\right)=\left(\begin{array}{l}
l_{y}^{(1)} \cdot L_{Z}^{(1)}-l_{z}^{(1)} \cdot L_{Y}^{(1)} \\
l_{z}^{(1)} \cdot L_{X}^{(1)}-l_{x}^{(1)} \cdot L_{Z}^{(1)} \\
l_{x}^{(1)} \cdot L_{Y}^{(1)}-l_{y}^{(1)} \cdot L_{X}^{(1)}
\end{array}\right) \\
& \sin \emptyset_{1}=\sqrt{\left(\bar{N}_{X}^{(1)}\right)^{2}+\left(\bar{N}_{Y}^{(1)}\right)^{2}+\left(\bar{N}_{Z}^{(1)}\right)^{2}} \\
& N_{2}(t)=\left(\begin{array}{l}
N_{X}^{(2)} \\
N_{Y}^{(2)} \\
N_{Z}^{(2)}
\end{array}\right)=\frac{1}{\sin \emptyset_{2}} \cdot\left|\begin{array}{ccc}
\mathrm{e}_{X} & \mathrm{e}_{Y} & \mathrm{e}_{Z} \\
l_{x}^{(2)} & l_{y}^{(2)} & l_{z}^{(2)} \\
L_{X}^{(2)} & L_{Y}^{(2)} & L_{Z}^{(2)}
\end{array}\right|= \\
& =\frac{1}{\sin \emptyset_{2}} \cdot\left(\begin{array}{l}
\bar{N}_{X}^{(2)} \\
\bar{N}_{Y}^{(2)} \\
\bar{N}_{Z}^{(2)}
\end{array}\right)
\end{aligned}
$$

where:

$$
\begin{aligned}
& \left(\begin{array}{l}
\bar{N}_{X}^{(2)} \\
\bar{N}_{Y}^{(2)} \\
\bar{N}_{z}^{(2)}
\end{array}\right)=\left(\begin{array}{l}
l_{y}^{(2)} \cdot L_{Z}^{(2)}-l_{z}^{(2)} \cdot L_{Y}^{(2)} \\
l_{z}^{(2)} \cdot L_{X}^{(2)}-l_{x}^{(2)} \cdot L_{z}^{(2)} \\
l_{x}^{(2)} \cdot L_{Y}^{(2)}-l_{y}^{(2)} \cdot L_{X}^{(2)}
\end{array}\right) \\
& \sin \emptyset_{2}=\sqrt{\left(\bar{N}_{X}^{(2)}\right)^{2}+\left(\bar{N}_{Y}^{(2)}\right)^{2}+\left(\bar{N}_{Z}^{(2)}\right)^{2}}
\end{aligned}
$$

Considering (6) - (7) the relationship (5) will have the following form:

$$
\begin{aligned}
& \boldsymbol{L}_{r}(t)=\left(\begin{array}{l}
N_{X}(t) \\
N_{Y}(t) \\
N_{Z}(t)
\end{array}\right)=\frac{1}{\sin \varnothing} \cdot\left|\begin{array}{ccc}
e_{X} & e_{Y} & e_{Z} \\
N_{X}^{(2)} & N_{y}^{(2)} & N_{z}^{(2)} \\
N_{X}^{(1)} & N_{Y}^{(1)} & N_{Z}^{(1)}
\end{array}\right|= \\
& =\frac{1}{\sin \varnothing} \cdot\left(\begin{array}{l}
\bar{N}_{X} \\
\bar{N}_{Y} \\
\bar{N}_{Z}
\end{array}\right)
\end{aligned}
$$

where:

$$
\begin{aligned}
& \left(\begin{array}{l}
\bar{N}_{X} \\
\bar{N}_{Y} \\
\bar{N}_{Z}
\end{array}\right)=\left(\begin{array}{l}
N_{y}^{(2)} \cdot N_{Z}^{(1)}-N_{Y}^{(1)} \cdot N_{z}^{(2)} \\
N_{X}^{(1)} \cdot N_{z}^{(2)}-N_{Z}^{(1)} \cdot N_{X}^{(2)} \\
N_{X}^{(2)} \cdot N_{Y}^{(1)}-N_{X}^{(1)} \cdot N_{y}^{(2)}
\end{array}\right) \\
& \sin \emptyset=\sqrt{\left.\left(\bar{N}_{X}\right)^{2}+\left(\bar{N}_{Y}\right)^{2}+\overline{(N}_{Z}\right)^{2}}
\end{aligned}
$$

Thus, at a time moment $t$ directional cosine angles of the SC R-bar in ICS are the functions of the measured values of the direction cosine angles of the vectors $\left(\boldsymbol{l}_{1}, \boldsymbol{l}_{2}\right)$ and $\left(\boldsymbol{L}_{1}, \boldsymbol{L}_{2}\right)$ that at this time moment determine stellar lines $\left({ }^{*} 1\right)$ and $\left({ }^{*} 2\right)$ in ST coordinate system and ICS correspondingly.

On the other hand, it is known, that at the same time moment $t$ the directional cosine angles of the SC R-bar in ICS are functions of the elements of keplerian orbit $\Omega, \mathrm{M}(t), i$ :

$$
L_{r}(t)=\left(\begin{array}{c}
\cos \Omega \cdot \cos M(t)-\sin \Omega \cdot \sin M(t) \cdot \cos i \\
\sin \Omega \cdot \cos M(t)+\cos \Omega \cdot \sin M(t) \cdot \cos i \\
\sin M(t) \cdot \cos i
\end{array}\right)
$$

By matching the relations (8) and (9) we'll get:

$$
\left(\begin{array}{l}
N_{X}(t) \\
N_{Y}(t) \\
N_{Z}(t)
\end{array}\right)=\left(\begin{array}{c}
\cos \Omega \cdot \cos M(t)-\sin \Omega \cdot \sin M(t) \cdot \cos i \\
\sin \Omega \cdot \cos M(t)+\cos \Omega \cdot \sin M(t) \cdot \cos i \\
\sin M(t) \cdot \sin i
\end{array}\right)
$$

As mean anomaly $\mathrm{M}(t)$ is time dependent unlike other keplerian orbit elements, let's express it through SC mean motion $n_{\mathrm{cp}}$ and plug it into equation set (10), then finally we'll get a set of self-contained equations:

$$
\begin{gathered}
\left(\begin{array}{l}
N_{X}(t) \\
N_{Y}(t)
\end{array}\right)= \\
=\left(\begin{array}{c}
\cos \Omega \cdot \cos \left(\mathrm{M}\left(t_{0}\right)+n_{\mathrm{cp}} \cdot\left(t-t_{0}\right)\right)-\sin \Omega \cdot \operatorname{ctg} i \cdot N_{Z}(t) \\
\sin \Omega \cdot \cos \left(\mathrm{M}\left(t_{0}\right)+n_{\mathrm{cp}} \cdot\left(t-t_{0}\right)\right)+\cos \Omega \cdot \operatorname{ctg} i \cdot N_{Z}(t)
\end{array}\right)
\end{gathered}
$$

with four unknown quantities: $\mathrm{M}_{0}\left(t_{0}\right), i, \Omega, n_{\mathrm{cp}}$. 


\section{CONCLUSIONS}

1. Minimal number of points from which sky images should be made is two. Moreover, each image should be processed to find the coordinates of at least two stars. In this case a set of four non-linear equations with four unknown quantities can be formed up. The set is solved by computational mathematics methods, for example Newton-Raphson method.

2. The processing of sky images made from three or more SC orbit points with determining the coordinates of at least two stars in each image makes it possible to solve the problem of SC autonomous navigation and attitude control by least squares method and assess the accuracy of the obtained results.

3. When three elements of SC keplerian orbit and its mean motion values have been determined by means of formulas well-known in ballistics, the values of SC R-bar and mean anomalies $\mathrm{M}\left(t_{1}\right), \mathrm{M}\left(t_{2}\right)$ can be found, as well as SC geo-centric coordinates and its component velocities in orbit trajectory points $t_{1}, t_{2}$.

\section{REFERENCES}

1. Прохоров М., Захаров А. (2011). Ориентация и навигация в космосе - новые методы и перспективы / Физика космоса: Труды 40-й Международной студенческой научной конференции // Екатеринбург. - Издательство Уральского университета. - Екатеринбург. - С. 170-195.

2. Урмаев М.С. Космическая фотограмметрия. (1989). М.: Недра, 1989. - 279 с.

3. Бахшиян Б. Ц., Федяев К. С. (2013). Основы космической баллистики и навигации: Курс лекций / М.: ИКИ РАН, серия «Механика, управление и информатика». - 119 с.

4. Lawrence A. (2012). Modern Inertial Technology: Navigation, Guidance, and Control, Springer.- 278 p. 